\title{
Targeted therapies: the answer to individualized treatment?
}

\author{
Lisa Hutchinson
}

Much excitement and optimism has been generated by the advent of targeted therapies for treating cancer. Notable examples include imatinib for treating chronic myelogenous leukemia and gastrointestinal tumors, gefitinib and erlotinib for non-small-cell lung cancers, trastuzumab in women with breast cancer who overexpress HER2, and sunitinib and sorafinib for renal cancer. Undoubtedly, these agents have contributed significantly to the armamentarium of approaches employed to treat and-in the adjuvant setting-cure cancer. Nonetheless, while tools and other technological advances developed in the past decades have fostered bed-to-benchside progress in clinical oncology, our understanding of the molecular carcinogenic process and the ability to test agents in multiple complex disease subtypes and settings, is, by comparison, still in its infancy.

Drugs developed to 'target' a specific kinase within the cell, for example, are usually characterized against only a handful (1-2\%) of the known kinases in humans; thus, the true 'specificity' of these agents is largely untested. While this does not hinder the rational design and testing of such drugs during clinical development, the extent to which other cellular targets are affected is unclear. Gene-expression profiling and molecular genetics have revealed that multiple clinically and biologically distinct molecular subtypes are present within the same tumor. As a result, we are beginning to determine how subgroups of patients respond to particular treatments on the basis of the genetic and epigenetic profile of their tumors, and we have begun to tailor the treatments accordingly. Given the biological diversity and continuing evolution of tumors, however, it is perhaps not surprising that a single treatment with a targeted therapy is unable to cure many patients. The redundancy of signaling pathways, crosstalk between multifunctional cellular signaling networks, and complex tissue microenvironment interactions mean

\section{Even with the sophisticated technologies available, we see only a snapshot of the dynamic carcinogenic process}

\section{Hutchinson is the Editor of Nature Clinical Practice Oncology.}

\section{Competing interests}

The author declared she has no competing interests.

www.nature.com/clinicalpractice doi:10.1038/ncponc0837 that the complete picture remains elusive. Even with the sophisticated technologies available, we see only a snapshot of the dynamic carcinogenic process.

Why and how cancer cells that initially respond to treatment become resistant is another mystery. We have only begun to scratch the surface in terms of our understanding of resistance. Admittedly, new insights have been gleaned from studies of gefitinib and imatinibmany of the patients initially responsive to these drugs developed resistance because they harbored secondary mutations in the drug target that interfered with the drug's binding or efficacy. Presumably, these secondary resistance mutations arose to circumvent the effects of the drug. Yet, not all patients who developed resistance acquired these mutations. Retrospective analyses have not been undertaken to assess the mutational response of patients treated with chemotherapy agents. It is clear that standard concepts in pharmacodynamics no longer account for either de novo or acquired drug resistance at the clinical level. If cancer cells develop multiple mutations in response to most treatments, the identification of 'treatment-response mutations' with targeted agents might also be misleading in the progress towards individualized treatment.

Exploring new drug combinations and dose schedules is one approach to overcome the problem of resistance with these new agents. Development of newer drugs that are active against a different portion of the 'target' are ongoing. But the testing of targeted drug combinations with adjuvant therapies and attaining optimum patient selection, pose an even bigger challenge for clinical trial testing. The number of patients needed to test such multiple drug combinations, especially with sufficient control arms, will be very difficult to ascertain and achieve. For now our optimism is justified, but targeted therapies used singly do not yet constitute an answer for individualized treatment. 